\title{
The relationship between WIG-subindexes: evidence from the Warsaw Stock Exchange
}

\section{Introduction}

Investors who are present in emerging markets are interested not only in returns, but also in the risk characteristics of stock prices in these markets. In particular, investors try to identify any signals of informational inefficiency in these markets in order to make larger profits. The existing contributions demonstrated that emerging markets are specific and their behavior is rather complex. This complexity depends on many factors. Empirical investigations on individual emerging markets make it easier to further understand the factors influencing returns and risk in these fast-growing markets. Stock indexes are frequently applied in testing for market efficiency. This is very important in cases when a high market concentration can be observed.

Most of the studies that examine the relationship among indexes use different indexes across countries. However, stock market indexes of different countries are subject to different monetary and fiscal policy shocks coming from their respective governments, as well as the specific structural problems in which each country may be confronted. Therefore, using data from a single country (e.g., Poland) enables us to eliminate the effects of different policy and structural shocks on stock market indexes. Thus, one may analyze the co-movements of stock market subindexes to assess the role of fundamentals in various sectors.

We believe that understanding the behavior and interaction among the subindexes will significantly contribute to the understanding of the role of major sectors of the Polish economy in shaping the behavior and efficiency of the WSE. In addition, an investigation of dependence among the major WIG index sec-

* AGH University of Science and Technology, Department of Applications of Mathematics in Economics, e-mail: henryk.gurgul@gmail.com

** Jagiellonian University in Cracow, Institute of Economics and Management, e-mail: robert.syrek@uj.edu.pl 
toral subindexes will be highly useful to individual and institutional investors who are keen on diversifying their portfolios by investing in the emerging Polish stock market. The non-existence of dependence among these sub-indexes enables the benefits of portfolio diversification among these indexed assets to be realized.

The findings of this research will help in answering some questions; e.g., do these subindexes behave similarly? Do they influence each other? Before answering these questions, we overview the selected contributions concerned with the dependence of subindexes in section 2 of this paper. In addition, we will also mention some applications of copulas to assess the relationships between financial variables (including stock indexes). The methodology used in this contribution is presented in section 3. The empirical results and their evaluation are provided in section 4. Section 5 concludes.

\section{Literature overview}

In one of the earliest contributions, Higgins (1988) assumed that an examination of links between stock market indexes may supply useful information in predicting future movement of stock prices, and even the economic performance of a country under study. In addition, there is evidence that the rejection of weak-form market efficiency could be explained by the high correlation among the dominant sector indexes (Arbelaez et al. 2001). Just in 1996, Ratner (1996) applied nine major equity indexes in order to examine the market efficiency of the Madrid Stock Exchange. He showed that index returns essentially departed from the random walk. Their distribution was non-normal.

Arbelaez et al. (2001) empirically examined the short-term and long-term linkages among the several stock indexes of the former Stock Exchange Medelin (now the Colombia Stock Exchange). They applied a methodology that included tests of stationarity, causality, cointegration, impulse response, and variance decomposition as well as a VEC model to a data set that covered daily prices for more than 7 years. The empirical findings supported the existence of dependence among the indexes analyzed.

Other researchers, taking into account index data from the Greek stock markets, made similar conclusions. The contributors came to the conclusion that the Athens Stock Exchange (ASE) is inefficient in a semi-strong sense. In the study by Kavussanos and Dockery (2001), multivariate generalizations and seemingly unrelated regressions are used. The results confirmed that the ASE is informationally inefficient. In other words, past stock prices have predictive power for future price movements. 
Siourounis (2002) used GARCH-type models and checked for their validity in the ASE Market. He showed that returns were correlated and that volatility exhibited autocorrelation. The author confirmed that the weak form of the efficientmarket hypothesis did not hold true in the ASE.

Niarchos and Alexakis (2003) found that specific price patterns existed. Moreover, the authors detected that trading rules based on these price patterns could be profitably exploited. They are superior with respect to a passive buy and hold strategy. So, this study also supplied evidence for the inefficiency of the ASE.

Panagiotidis (2005) rejected the random-walk hypothesis for the three different FTSE/ASE indexes after the introduction of the Euro.

Patra and Poshakwale (2008) reported that the Athens Stock Exchange has six sectors: namely, Banking, Industrial, Construction, Insurance, Investment, and Holding. They account for more than $63 \%$ of the total market capitalization. The authors provided empirical evidence on the short-term and long-term relationships among these major stock subindexes of the ASE. The contributors suggested that the sector indexes do not show a consistent and strong longterm relationship. However, the banking sector can have a strong influence on the returns and volatility of other sectors - at least in the short-run. The variance of returns for most sectors is largely stimulated by their own innovations. However, the banking sector can explain around $25 \%$ of variance of the construction sector, and the insurance sectors around 15\% of the variance of the industrial, investment, and holding sectors. From this research came the predominant role of the banking sector (subindex). Thus, the banking sector index could have predictive power for short-term movements in other sector indexes. This means that the ASE is not efficient in the sense of its weak-form efficiency.

It is important to note that investors need to understand the links between the various indicators. Moreover, much of the known contributions focused on the transfer of information and dealt mostly with advanced economies, followed by newly-liberated economies in South Asia and Latin America.

However, due to the impact of the latest global crises and imbalances caused on the economies of emerging markets in these two regions, investors had to show into other emerging markets, such as the markets of the Middle East and North Africa, as those markets are characterized by high returns and volatility, low correlation with world markets, and volatility clustering (see Lagoarde-Segot and Lucey 2006). Such a type of research allows us to discover the degree of interdependence and the nature of information flow across sectors, as well as the relative importance of these sectors in explaining variations of returns in these sectors. A study of this type attempts to fill the gap of examining the transmission of information across sectors in the same stock market (Wang et al. 2005). 
Also, research concerned with the interdependence of domestic sectoral subindexes from CEE countries like Poland is, to the best of our knowledge, not reflected in the available literature on dependencies between sectoral subindexes. In contrast to the reviewed contributions, we will conduct our analysis of the relationship among Polish sector subindexes by regular vine copulas.

In the last decade, copulas became a very popular tool in the modeling of dependencies between financial variables, especially after the outbreak of the global financial crisis (GFC) in 2008. Extensive contributions to the copula theory are available in Joe (1997) and Nelsen (2006). However, these monographs focus on two-dimensional copulas, developed to reflect links between two variables.

In order to analyze links between more variables, the hierarchical, copulabased structures have recently been used in some new developments in multivariate modelling. In the set of these structures is the pair-copula construction (PCC), initiated by Joe (1996). Its properties were proven by Bedford and Cooke $(2001,2002)$ as well as by Kurowicka and Cooke (2006). Aas et al. (2009) formulated a mathematical background and interpretation of this class of copulas. As a consequence, the PCC was used in numerous applications (Chollete et al. 2009, Berg and Aas 2009).

The applications of copulas in the time series models (e.g., Patton 2009, and the COPAR model of Brechmann and Czado 2014) used a vector autoregressive VAR model for analyzing the non-linear and asymmetrical co-dependencies between two series. More recent applications of a novel and recently-developed method of statistical and mathematical analysis to the assessment of financial risk (namely, regular vine copulas) are presented in Allen et al. (2013).

In our paper, we focus on the static modeling of dependencies based on R Vines in the context of modeling the co-dependencies of Polish WIG-subindexes.

The rest of the article is organized as follows: the next section describes the methodology applied. The fourth chapter contains the described dataset and subsequent results. The last section concludes the paper.

\section{Methodology: regular vine copulas}

The vines introduced by Bedford and Cooke $(2001,2002)$ are a graphical representation of a special class of multivariate distributions based on simple building blocks called pair-copulas (Aas et al. 2009). They are described in more detail in Kurowicka and Cooke (2006) as well as in Kurowicka and Joe (2011). 


\subsection{Regular vine copula}

A regular vine on $\mathrm{n}$ elements is a nested set of $n-1$ trees (connected acyclical graphs) such that the edges of tree $j$ become the nodes of tree $j+1$. The set on nodes in the first tree contains all $n$ indexes, while the set of edges is a set of $n-1$ pairs of these indexes. The second tree is constructed so that the set of nodes contains a set of pairs of all indexes, and the set of edges is built of pairs of pairs of indexes, etc. Formally, the definition of a regular vine (R-vine) is as follows (see Dißmann et al. 2013):

\section{Definition 1}

$\boldsymbol{V}=\left(\boldsymbol{T}_{1}, \ldots, \boldsymbol{T}_{n-1}\right)$ is an R-vine on $n$ elements if

1. $T_{1}$ is a tree with nodes $N_{1}=\{1, \ldots, n\}$ and a set of edges denoted $E_{1}$.

2. For $i=2, \ldots, n-1, T i$ is a tree with nodes $N i=E_{i-1}$ and edge set $E_{i}$.

3. For $i=2, \ldots, n-1$ and $\{a, b\} \in E_{i}$ with $a=\left\{a_{1}, a_{2}\right\}$ and $b=\left\{b_{1}, b_{2}\right\}$ it must hold that $\#(a \cap b)=1$

The last condition (called the proximity condition) ensures that there is an edge connecting two nodes in tree $T_{j+1}$ if these nodes share a common node in tree $T_{j}$. The proximity condition allows us to identify each edge $e=\left(a_{e}, b_{e} \mid D_{e}\right)$, where $\{a e, b e\}$ is the conditioned set and $D e$ is conditioning set of edge $e$ Here $a_{e}, b_{e} \in\{1, \ldots, n\}, a_{e} \neq b_{e}$, and $D_{e} \subset\{1, \ldots, n\}$. In Dißmann et al. (2013), Bedford and Cooke (2002) reader can find more details.

An $n$ - dimensional regular vine copula is based on the R-vine structure and is used to specify bivariate copulas in PCC, pair-copula construction (Aas 2009). A formal definition of the R-vine copula specification is as follows (Dißmann et al. 2013, Czado et al. 2013).

\section{Definition 2}

$(F, V, B)$ is an R-vine copula specification if $F=\left(F_{1}, \ldots, F_{n}\right)$ is a vector of continuous invertible distributions functions, $V$ is a an $n$-dimensional R-vine and $B=\left\{B_{e} \mid i=1, \ldots, n-1 ; e \in E_{i}\right\}$ is a set of copulas with $B_{e}$ being a bivariate copula, a so-called pair-copula.

Specifying $n-1$ unconditional bivariate copulas between variables indexed by the conditioned sets of the edges constructs the first tree of the R-vine copula.

In the second tree of the R-vine, the bivariate copulas between variables indexed by a conditioned set are specified. They are conditional on variables indexed by the conditioning sets of edges of the R-vine.

The density of the R-vine copula specification was shown in the following theorem by Bedford and Cooke (2001, 2002). 


\section{Theorem}

Let $(F, V, B)$ be an R-vine copula specification on $n$ elements. There is a unique $n$-dimensional distribution $\mathrm{F}$ that realizes this R-vine copula specification with density:

$$
f_{1, \ldots, n}(\boldsymbol{x})=\prod_{k=1}^{n} f_{k}\left(x_{k}\right) \prod_{i=1}^{n-1} \prod_{e \in E_{i}} c_{a_{e}, b_{e} \mid D_{e}}\left(F_{a_{e} \mid D_{e}}\left(x_{a_{e}} \mid \boldsymbol{x}_{D_{e}}\right), F_{b_{e} \mid D_{e}}\left(x_{b_{e}} \mid \boldsymbol{x}_{D_{e}}\right)\right)
$$

where $x=\left(x_{1}, \ldots, x_{n}\right)^{\prime}, e=a_{e}, b_{e} \mid D_{e}$ and $x_{D_{e}}$ contains the variables in $D_{e}$, i.e. $\boldsymbol{x}_{\boldsymbol{D}_{e}}=\left\{\boldsymbol{x}_{\boldsymbol{i}} \mid \boldsymbol{i} \in \boldsymbol{D}_{\boldsymbol{e}}\right\}$. Here $\boldsymbol{F}_{\boldsymbol{a}_{e} \mid \boldsymbol{D}_{e}}$ denotes the conditional distribution function of $\boldsymbol{X}_{\boldsymbol{a}_{e}}$ given $\boldsymbol{X}_{D_{e}}=\boldsymbol{x}_{\boldsymbol{D}_{e}}$ when $\mathrm{X}=\left(X_{1}, \ldots, X_{n}\right)^{\prime}$ has distribution $\mathrm{F}$.

\subsection{Selecting and estimating regular vine copula}

The fitting R-vine copula specification to dataset is performed in the following steps:

1. Estimation of parameters of margins,

2. Selection of proper R-vine structure,

3. Choosing bivariate copulas,

4. Estimation of parameters of chosen copulas.

In the case of a time series, appropriate ARMA-GARCH class models are fitted to a given dataset at first. Standardized residuals and their transformation to uniform are used in the next steps. Since the numbers of possible R-vines increases very rapidly (Morales-Napoles et al. 2010), Dißmann et al. (2013) developed a sequential method to select the tree structure of the R-vine. They proceed sequentially, starting by defining the first tree for the R-vine, continuing with the second tree, etc. The trees are selected in such a way that the chosen pairs model the strongest pairwise dependencies that are present. In other words, they select the spanning tree that maximizes the sum of absolute empirical Kendall's taus:

$$
\max \sum_{e=\{j, k\}}\left|\widehat{\tau}_{j, k}\right|
$$

By AIC criterion, a bivariate copula is selected, and in the next step, a likelihood estimation method is used to estimate the parameters of pair-copulas. During the selection, the independence test based on Kendall's tau (Genest and Favre 2007) is performed. If there is no reason to reject the null, the independence copula is chosen, and no further steps are taken. 


\subsection{Matrix representation of R-vines}

Because storage of the structure of an R-vine is very expensive, there is a need to find an easy way to develop statistical inference algorithms. Morales-Napoles (2008) uses a lower triangular matrix to store R-vine and to encode corresponding pair-copulas and their parameters. In Dißmann et al. (2013), one can find matrix representation of R-vines. We follow their definitions. Let $M=\left(m_{i, j}\right)_{i, j=1, \ldots, n}$ be a lower triangular matrix. For $i=1, \ldots, n-1$ two sets are defined in following way:

$$
\begin{gathered}
B_{M}(i):=\left\{\left(m_{i, i}, D\right) \mid k=i+1, \ldots, n ; D=\left\{m_{k, i}, \ldots, m_{n, i}\right\}\right\} \\
\tilde{B}_{M}(i):=\left\{\left(m_{k, i}, D\right) \mid k=i+1, \ldots, n ; D=\left\{m_{i, i}\right\} \cup\left\{m_{k+1, i}, \ldots, m_{n, i}\right\}\right\}
\end{gathered}
$$

The definition of the R-vine matrix that codes all information about R-vine structure is as follow.

\section{Definition 3}

A lower triangular matrix $M=\left(m_{i, j}\right)_{i, j=1, \ldots, n}$ is called an R-vine matrix if for $i=1, \ldots, n-1$ and for all $k=i+1, \ldots, n-1$ there is a $j$ in $i=1, \ldots, n-1$ with:

$$
\left(m_{k, i},\left\{m_{k+1, i} \ldots, m_{n, i}\right\}\right) \in B_{M}(i) \text { or } \in \tilde{B}_{M}(i)
$$

In terms of elements of matrix $M$, it is possible to evaluate the joint density of a regular vine (for further details see Dißmann et al. 2013) which is used in the algorithm that fully describes the parametric form of R-vine distribution.

$$
f_{1, \ldots, n}=\prod_{j=1}^{n} f_{j} \prod_{k=n-1}^{1} \prod_{i=n}^{k+1} c_{m_{k, k}, m_{i, k} \mid m_{i+1, k, \ldots,}, m_{n, k}}\left(F_{m_{k, k}, m_{i, k} \mid m_{i+1, k, \ldots,} m_{n, k}}, F_{m_{i, k}, m_{i, k} \mid m_{i+1, k, \ldots,} m_{n, k}}\right)
$$

Additional information about the types of pair-copulas and their parameters are contained in matrices $T=\left(t_{i, j}\right)_{i, j=1, \ldots, n}$ and $P=\left(p_{i, j}\right)_{i, j=1, \ldots, n}$, respectively. In Dißmann et al. (2013), one can find examples of matrix representations of given R-vines, likelihood computation, and sampling procedures.

\section{The data and estimation results}

The WSE calculates the following WIG index sectoral subindexes: WIG-banking (since December 31, 1998), WIG-construction (since December 31, 1998), 
WIG-chemicals (since September 19, 2008), WIG-developers (since June 15, 2007), WIG-energy (since December 31, 2009), WIG-IT (since December 31,1998), WIG-media (since December 31, 2004), WIG-oil \& gas (since December 31, 2005), WIG-food (since December 31, 1998), WIG-basic materials (since December 31, 2010), and WIG-telecom (since December 31, 1998). Modifications in all exchange index portfolios are made quarterly following the trading session on the third Fridays of March, June, September, and December. A periodic modification announcement is made one week before change implementation (i.e., prior to the third Fridays in March, June, September and December).

At the baseline date, the values of all subindexes under study equalled the value of the WIG index. The values of subindexes allow us to assess the efficiency of investments into businesses in various sectors of the economy. In the subindex portfolios, there are the same weightings as in the WIG index portfolio, but selected based on sectoral criterion. The methodology of the compilation of sectoral subindexes refers to WIG index methodology, and accounts for income from dividend and subscription rights.

We consider daily closing prices of 11 sector indexes from WSE (WIG-banking, WIG-construction, WIG-chemicals, WIG-developers, WIG-energy, WIG-IT, WIG-media, WIG-oil\&gas, WIG-food, WIG-basic materials, and WIG-telecom). The dataset covers the period from 2011.03.01 to 2014.07.21 and contains 844 observations. We compute the continuous (logarithmic) returns:

$$
r_{t}=100 \cdot \log \frac{p_{t}}{p_{t-1}}
$$

where $p_{t}$ is the price index at the time t. In Table 1 we present descriptive statistics of logarithmic returns under study.

Table 1

Summary statistics of logarithmic returns

\begin{tabular}{|c|l|c|c|c|c|}
\hline Number & \multicolumn{1}{|c|}{ Sector } & Mean & Std. dev. & Skewness & Kurtosis \\
\hline 1 & WIG-banking & 0.0222 & 1.4478 & -0.4438 & 6.9373 \\
\hline 2 & WIG-construction & -0.1099 & 1.4512 & -0.9466 & 7.1724 \\
\hline 3 & WIG-chemicals & 0.0706 & 1.7922 & -0.5833 & 6.6821 \\
\hline
\end{tabular}


The relationship between WIG-subindexes: evidence from the Warsaw Stock Exchange

Table 1 cont.

\begin{tabular}{|c|l|c|c|c|c|}
\hline 4 & WIG-developers & -0.0671 & 1.3942 & -0.6603 & 7.0649 \\
\hline 5 & WIG-energy & 0.0022 & 1.3383 & -0.3637 & 6.1903 \\
\hline 6 & WIG-IT & -0.0040 & 1.2876 & -0.5224 & 5.9120 \\
\hline 7 & WIG-media & -0.0002 & 1.4228 & -0.1354 & 4.3137 \\
\hline 8 & WIG-oil\&gas & 0.0054 & 1.6132 & -0.3635 & 5.7829 \\
\hline 9 & WIG-food & -0.0747 & 1.5416 & -0.5995 & 8.0749 \\
\hline 10 & WIG-basic materials & -0.0143 & 1.9237 & -0.8616 & 6.9023 \\
\hline 11 & WIG-telecom & -0.0257 & 1.7445 & -5.7214 & 87.1280 \\
\hline
\end{tabular}

Negative skewness and high kurtosis suggest that normal distribution should not be used to model these time series. These suggestions are confirmed with the Jarque-Bera test for normality (not reported here). Very high values of these statistics for WIG-telecom are caused by a rapid price fall in 2013.02.12 (from 1067.89 to 805.51$)$.

\subsection{Models for margins}

In order to capture possible autocorrelation and heteroscedasticity in a time series, we apply ARMA models and GARCH class models (GARCH, TARCH, or GJR). The Skewed- $t$ distribution in chosen to describe error terms. We follow the proposition of Fernandez and Steel (1998). They introduced skewness into unimodal and symmetric distributions by applying inverse scale factors. Given a skewness parameter $\lambda$, the density of random variable $z$ is:

$$
f(z \mid \lambda)=\frac{2}{\lambda+\lambda^{-1}}\left[f(\lambda z) H(-z)+f\left(\lambda^{-1} z\right) H(z)\right]
$$

where $\lambda \in \mathbb{R}^{+}$and $H(\cdot)$ is the Heaviside function. 
We use R package rugarch to obtain estimation results of models for margins (AR-TGARCH, with few exceptions). They are placed in the table below (robust standard errors in brackets). We use numeration of indexes from Table 2.

Table 2

Estimation results of ARMA-GARCH class models

\begin{tabular}{|c|c|c|c|c|c|c|c|c|}
\hline $\begin{array}{c}\text { Num- } \\
\text { ber }\end{array}$ & $\varphi \mathbf{1}$ & $\varphi 2$ & $\omega$ & $\alpha$ & $\beta$ & $\gamma$ & $\lambda$ & $v$ \\
\hline 1 & - & - & $\begin{array}{c}0.0149 \\
(0.0065)\end{array}$ & $\begin{array}{c}0.0650 \\
(0.0126)\end{array}$ & $\begin{array}{c}0.9387 \\
(0.0107)\end{array}$ & $\begin{array}{c}0.5256 \\
(0.1728)\end{array}$ & $\begin{array}{c}0.8965 \\
(0.0455)\end{array}$ & $\begin{array}{c}12.5685 \\
(4.558)\end{array}$ \\
\hline 2 & $\begin{array}{l}0.1627 \\
(0.036)\end{array}$ & $\begin{array}{c}0.0700 \\
(0.0336)\end{array}$ & $\begin{array}{c}0.1395 \\
(0.0715)\end{array}$ & $\begin{array}{c}0.1197 \\
(0.0418)\end{array}$ & $\begin{array}{c}0.8045 \\
(0.0764)\end{array}$ & $\begin{array}{c}0.5745 \\
(0.2059)\end{array}$ & $\begin{array}{c}0.9493 \\
(0.0445)\end{array}$ & $\begin{array}{c}5.6141 \\
(1.0661)\end{array}$ \\
\hline 3 & $\begin{array}{c}0.1282 \\
(0.0313)\end{array}$ & $\begin{array}{l}-0.1015 \\
(0.0278)\end{array}$ & $\begin{array}{c}0.1916 \\
(0.0808)\end{array}$ & $\begin{array}{l}0.1895 \\
(0.047)\end{array}$ & $\begin{array}{l}0.7464 \\
(0.074)\end{array}$ & $\begin{array}{c}0.3190 \\
(0.1122)\end{array}$ & $\begin{array}{c}0.9041 \\
(0.0428)\end{array}$ & $\begin{array}{c}6.8732 \\
(1.6006)\end{array}$ \\
\hline 4 & $\begin{array}{c}0.1053 \\
(0.0347)\end{array}$ & - & $\begin{array}{c}0.0405 \\
(0.0211)\end{array}$ & $\begin{array}{c}0.0738 \\
(0.0248)\end{array}$ & $\begin{array}{c}0.9111 \\
(0.0312)\end{array}$ & $\begin{array}{c}0.3414 \\
(0.1668)\end{array}$ & $\begin{array}{c}0.9349 \\
(0.0486)\end{array}$ & $\begin{array}{c}7.4408 \\
(1.7025)\end{array}$ \\
\hline 5 & - & - & $\begin{array}{c}0.0530 \\
(0.0301)\end{array}$ & $\begin{array}{c}0.0840 \\
(0.0243)\end{array}$ & $\begin{array}{c}0.8891 \\
(0.0332)\end{array}$ & - & $\begin{array}{c}0.9405 \\
(0.0444)\end{array}$ & $\begin{array}{c}6.6700 \\
(1.5705)\end{array}$ \\
\hline 6 & - & - & $\begin{array}{c}0.0428 \\
(0.0219)\end{array}$ & $\begin{array}{c}0.0787 \\
(0.0266)\end{array}$ & $\begin{array}{c}0.9072 \\
(0.0332)\end{array}$ & $\begin{array}{c}0.6141 \\
(0.2184)\end{array}$ & $\begin{array}{c}0.9319 \\
(0.0407)\end{array}$ & $\begin{array}{l}5.6480 \\
(1.103)\end{array}$ \\
\hline 7 & $\begin{array}{c}0.0903 \\
(0.0362)\end{array}$ & - & $\begin{array}{c}0.1100 \\
(0.0565)\end{array}$ & $\begin{array}{c}0.1186 \\
(0.0322)\end{array}$ & $\begin{array}{c}0.8282 \\
(0.0593)\end{array}$ & $\begin{array}{c}0.3127 \\
(0.1464)\end{array}$ & $\begin{array}{c}0.9633 \\
(0.0468)\end{array}$ & $\begin{array}{c}11.3866 \\
(4.253)\end{array}$ \\
\hline 8 & - & - & $\begin{array}{c}0.0486 \\
(0.0206)\end{array}$ & $\begin{array}{c}0.0375 \\
(0.0146)\end{array}$ & $\begin{array}{c}0.9405 \\
(0.0186)\end{array}$ & $\begin{array}{c}0.9865 \\
(0.4428)\end{array}$ & $\begin{array}{c}1.0352 \\
(0.0466)\end{array}$ & $\begin{array}{c}6.9573 \\
(1.5133)\end{array}$ \\
\hline 9 & - & - & $\begin{array}{c}0.2439 \\
(0.1182)\end{array}$ & $\begin{array}{c}0.1207 \\
(0.0428)\end{array}$ & $\begin{array}{c}0.7762 \\
(0.0807)\end{array}$ & - & $\begin{array}{l}0.9589 \\
(0.044)\end{array}$ & $\begin{array}{c}6.4246 \\
(1.3272)\end{array}$ \\
\hline 10 & $\begin{array}{c}0.0662 \\
(0.0292)\end{array}$ & - & $\begin{array}{l}0.0394 \\
(0.024)\end{array}$ & $\begin{array}{c}0.0561 \\
(0.0167)\end{array}$ & $\begin{array}{c}0.9357 \\
(0.0225)\end{array}$ & $\begin{array}{c}0.5579 \\
(0.2472)\end{array}$ & $\begin{array}{l}0.8680 \\
(0.041)\end{array}$ & $\begin{array}{c}6.7098 \\
(1.3493)\end{array}$ \\
\hline 11 & - & - & $\begin{array}{c}0.0580 \\
(0.0385)\end{array}$ & $\begin{array}{c}0.1290 \\
(0.0505)\end{array}$ & $\begin{array}{c}0.9100 \\
(0.0334)\end{array}$ & $\begin{array}{l}-0.1021 \\
(0.0433)\end{array}$ & $\begin{array}{c}0.9853 \\
(0.0371)\end{array}$ & $\begin{array}{c}3.2372 \\
(0.3731)\end{array}$ \\
\hline
\end{tabular}


In the case of WIG-energy and WIG-food, the GARCH model was estimated, while for the WIG-telecom model, GJR. In all of the remaining cases, the TARCH model was chosen to describe conditional volatility. In the model of conditional mean WIG-basic materials, $\varphi_{1}$ is in fact the MA(1) model coefficient. We check the goodness-of-fit by using Ljung-Box and ARCH tests applied to standardized residuals. We transform standardized residuals to uniform by using estimated parameters of conditional distribution, and finally we apply the Anderson-Darling goodness-of-fit test. In all cases, there is no reason to reject the null that transformed residuals are i.i.d.

\subsection{R-vine copula model}

In the next step, we use transformed standardized residuals to estimate the dependence structure described by the R-vine copula. For this purpose, we use the R-package VineCopula. The appropriate pair-copula families are estimated using the maximum likelihood estimation method and selected by the AIC criterion. We try elliptical copulas, one- and two-parameter Archimedean copulas, along with their survival versions. Trees of the R-vine copula are selected using maximum spanning trees with absolute values of pairwise correlation to Kendall's coefficients as weights (Dißmann et al. 2013). According to the Dißmann et al. (2013) notation presented in the previous section we get matrix $M$ that codes the structure represented by the estimated R-vines copula.

$$
M=\left[\begin{array}{ccccccccccc}
2 & & & & & & & & & & \\
11 & 6 & & & & & & & & & \\
5 & 11 & 9 & & & & & & & & \\
3 & 5 & 11 & 7 & & & & & & & \\
8 & 3 & 5 & 11 & 4 & & & & & & \\
10 & 8 & 3 & 5 & 11 & 10 & & & & & \\
6 & 10 & 8 & 3 & 5 & 11 & 3 & & & & \\
9 & 9 & 10 & 8 & 3 & 5 & 11 & 5 & & & \\
7 & 7 & 4 & 10 & 8 & 3 & 5 & 11 & 8 & & \\
1 & 4 & 7 & 4 & 10 & 8 & 1 & 1 & 11 & 11 & \\
4 & 1 & 1 & 1 & 1 & 1 & 8 & 8 & 1 & 1 & 1
\end{array}\right]
$$

The matrix of pair-copula families $T$ associated with matrix $M$ is as follows: ( 0 stands for independence copula, 1 for normal, 2 for $t$ copula, 4 for Gumbel, 
5 for Frank, 6 for Joe, 7 for BB1, 14 for survival Gumbel and 17 for survival BB1, see Nelsen (2006) for details).

$$
\boldsymbol{T}=\left[\begin{array}{cccccccccc}
0 & & & & & & & & & \\
0 & 0 & & & & & & & & \\
5 & 3 & 0 & & & & & & & \\
0 & 0 & 4 & 1 & & & & & & \\
3 & 5 & 5 & 5 & 0 & & & & & \\
4 & 1 & 5 & 1 & 0 & 0 & & & & \\
2 & 5 & 1 & 14 & 5 & 14 & 0 & & & \\
1 & 5 & 4 & 4 & 5 & 5 & 5 & 6 & & \\
5 & 2 & 1 & 1 & 1 & 1 & 2 & 1 & 5 & \\
14 & 17 & 14 & 2 & 7 & 2 & 2 & 7 & 17 & 1
\end{array}\right]
$$

Similar matrices of pair copula parameters associated with matrix $M$ could be presented (they are available from the authors upon request). Finally, in Figure 1 we present the first tree of the R-vine structure. The edges are labeled with names of pair-copulas and theoretical Kendall's taus coefficients in respect to the estimated copulas.

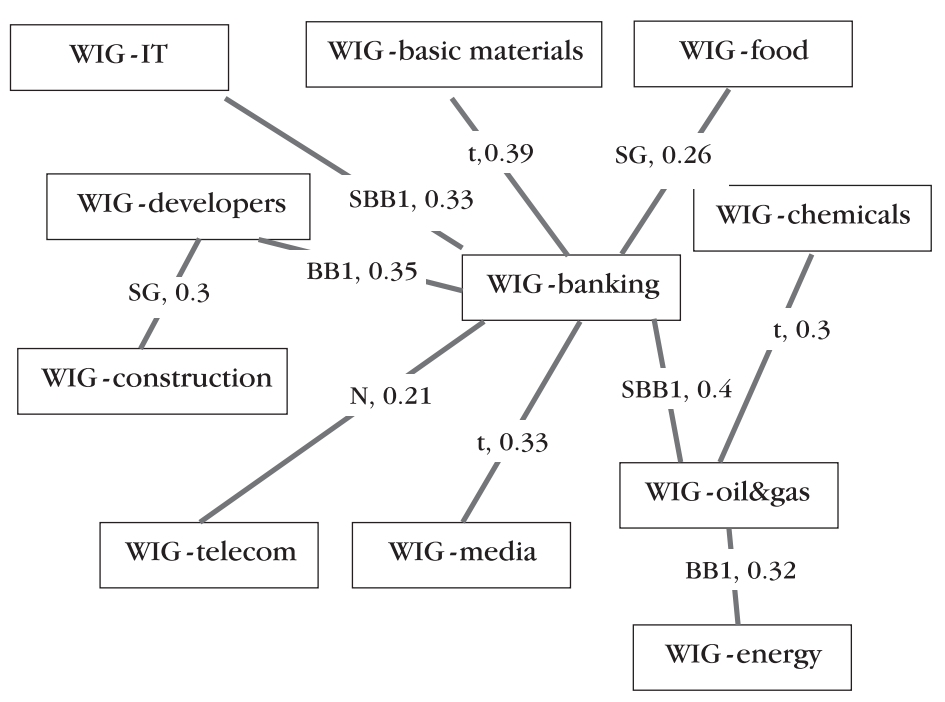

Figure 1. First tree of estimated R-vine copula 
Analyzing the matrices and Figure 1, we can make some conclusions. The sector WIG-banking is related to 6 of 11 sectors. This is not the case for the WIGchemical, WIG-energy, or WIG-construction sectors. The sector WIG-chemicals and WIG-energy more strongly depend on WIG-oil \& gas than on sector WIG-banking. Similarly, the dependence of sector WIG-construction is not directly related to WIG-banking, but it is related via sector WIG-developers. In most cases, dependence between sectors is described by copulas with tail-dependence coefficients. In some of them, tail dependence is symmetrical (described by $t$-copula or normal copula). The table below (Tab. 3) contains tail-dependence coefficients according to the estimated copulas of the first tree.

Table 3

Tail dependence coefficients of pair-copulas of first tree

\begin{tabular}{|l|c|c|}
\hline \multicolumn{1}{|c|}{ Pair of indices } & $\tau_{U}$ & $\tau_{L}$ \\
\hline WIG-banking - WIG-basic materials & 0.0572 & 0.0572 \\
\hline WIG-banking - WIG-media & 0.0854 & 0.0855 \\
\hline WIG-banking - WIG-food & 0 & 0.3353 \\
\hline WIG-banking - WIG-IT & 0.0115 & 0.3651 \\
\hline WIG-banking - WIG-oil\&gas & 0.2199 & 0.3763 \\
\hline WIG-banking - WIG-developers & 0.2589 & 0.2996 \\
\hline WIG-banking - WIG-telecom & 0 & 0 \\
\hline WIG-construction - WIG- developers & 0 & 0.3707 \\
\hline WIG-oil\&gas - WIG-energy & 0.1888 & 0.2665 \\
\hline WIG-oil\&gas - WIG-chemicals & 0.0424 & 0.0425 \\
\hline
\end{tabular}

In the case of copulas with asymmetrical tail dependence in the tails, lower tail dependence coefficients are greater than upper tail dependence coefficients. Extremely low logreturns are more dependent that extremely high. This pattern is often observed in financial time series.

\section{Conclusions}

The aim of this paper s to show the practical usefulness of regular vines copula in the description of structures of dependencies between 11 WIG-sectoral subindexes from Warsaw Stock Exchange. In general, the existence of dependence between subindexes means that fluctuations of prices in one stock subindex can 
be determined or predicted to some extent using a part of the information set provided by the other stock subindexes.

Our study indicated the central role of the WIG-banking sector in the economy and its strong influence on the other subsectors. The leading role of the WIGbanking sector implies that changes in this sector subindex could potentially be used in predicting movement in other WIG-subindexes. In this sense, the situation in the banking sector determines the possibility of financing other sectors, and therefore, facilitating growth of these sectors.

The predominant role of the banking sector in the WSE could be explained by its significance in the Polish economic system. The Polish banking sector subindex includes 13 banks. These 13 banks are dominant players in the WSE, with a combined market capitalization value of 325,52 billion zlotys (more than a $50 \%$ share in capitalization of the 11 subsectors under study) and over a $40 \%$ share of total market capitalization of the WSE in 2013. Thus, higher market capitalization and greater liquidity of the banking sector stocks is reflected in the leading influence of the banking sector on the other subindexes reported in our analysis in the previous sections.

In addition, it follows from our study that this influence is asymmetrical. The dependence between WIG-banking and other subindexes is more pronounced in the lower tail of the returns under study. In other words, the links between WIG-banking and the remaining WIG-subindexes are stronger in the bear phase of the Warsaw Stock Exchange.

\section{Acknowledgements}

Financial support for this paper from the National Science Centre of Poland (Research Grant DEC-2012/05/B/HS4/00810) is by first author gratefully acknowledged. We would like to thank the two anonymous referees for valuable comments and suggestions on earlier versions of this paper.

\section{References}

[1] Aas, K., Czado, C., Frigessi, A. and Bakken, H. (2009) 'Pair-copula constructions of multiple dependence', Insurance: Mathematics and Economics, vol. 44 (2), pp. 182-198.

[2] Allen, D.E., Ashra, M.A., McAleer, M., Powell, R.J. and Singh, A.K. (2013) 'Financial Dependence Analysis: Applications of Vine Copulas', Statistica Neerlandica, vol. 67 (4), pp. 403-435.

[3] Arbeláez, H., Urrutia, J. and Abbas, N. (2001) 'Short-term and long-term linkages among the Colombian capital market indexes', International Review of Financial Analysis, vol. 10 (3), pp. 237-273. 
[4] Bedford, T. and Cooke, R.M. (2001) 'Probability density decomposition for conditionally dependent random variables modeled by vines', Annals of Mathematics and Artificial intelligence, vol. 32, pp. 245-268.

[5] Bedford, T. and Cooke, R.M. (2002) 'Vines - a new graphical model for dependent random variables', Annals of Statistics, vol. 30, pp. 1031-1068.

[6] Berg, D. and Aas, K. (2009) 'Models for construction of higher-dimensional dependence: A comparison study', European Journal of Finance, vol. 15, pp. 639-659.

[7] Chollete, L., Heinen, A. and Valdesogo, A. (2009) 'Modeling international financial returns with a multivariate regime switching copula', Journal of Financial Econometrics, vol. 7, pp. 437-480.

[8] Brechmann, E. and Czado, C. (2014) 'Risk management with high-dimensional vine copulas: An analysis of the Euro Stoxx 50', Statistics \& Risk Modeling, vol. 30 (4), pp. 307-342.

[9] Brechmann, E.C. and Czado, C. (2014) 'COPAR-Multivariate Time Series Modeling Using the Copula Autoregressive Model', Applied Stochastic Models in Business and Industry, DOI: 10.1002/asmb.2043.

[10] Chollete, L., Heinen, A. and Valdesogo, A. (2009) 'Modeling international financial returns with a multivariate regime-switchng copula', Journal of Financial Econometrics, vol. 7 (4), pp. 437-480.

[11] Dißmann, J.F., Brechmann, E.C., Czado, C. and Kurowicka, D. (2013) 'Selecting and estimating regular vine copulae and application to financial returns', Computational Statistics \& Data Analysis, vol. 59 (1), pp. 52-69

[12] Higgins, B. (1988) 'Is a recession inevitable this year?', Economic Review, vol. 73 (1), Federal Reserve Bank of Kansas City, pp. 3-16.

[13] Joe, H. (1996) 'Families of m-variate distributions with given margins and $\mathrm{m}(\mathrm{m}-1) / 2$ bivariate dependence parameters', in Rueschendorf, L., Schweizer B. and Taylor M.D. (ed.) Distributions with fixed marginals and related topics, Hayward: Institute of Mathematical Statistics, pp. 120-141.

[14] Joe, H. (1997) Multivariate Models and Dependence Concepts, London: Chapman and Hall.

[15] Kavussanos, M.G. and Dockery, E. (2001) 'A multivariate test for stock market efficiency: the case of ASE', Applied Financial Economics, vol. 5, pp. 573-584.

[16] Kurowicka, D. and Cooke, R.M. (2006) Uncertainty Analysis with High Dimensional Dependence Modelling, Chichester: John Wiley.

[17] Kurowicka, D. and Joe, H. (eds) (2011) Dependence Modeling: Vine Copula Handbook. Singapore: World Scientific Publishing Co.

[18] Lagoarde-Segot, T. and Lucey, B. (2006) Efficiency in emerging marketsEvidence from the MENA region. International Financial Markets. Institutions and Money, vol. 18, pp. 94-105.

[19] Morales-Nápoles, O., Cooke, R. and Kurowicka D. (2010) About the number of vines and regular vines on $\mathrm{n}$ nodes. Preprint. 
[20] Nelsen, R. (2006) An introduction to copulas, New York: Springer.

[21] Niarchos N.A. and Alexakis C.A. (2003) 'Intraday stock price patterns in the Greek stock exchange', Applied Financial Economics, vol. 1, pp. 13-22.

[22] Patra, T. and Poshakwale, S.S. (2008) 'Long-run and short-run relationship between the main stock indexes: evidence from the Athens stock exchange', Applied Financial Economics, vol. 18, pp. 1401-1410.

[23] Panagiotidis, T. (2005) 'Market capitalisation and efficiency. Does it matter? Evidence from the Athens stock exchange', Applied Financial Economics, vol. 15, pp. 707-713.

[24] Patton, A.J. (2009) 'Copula-based models for financial time series', in Andersen, T.G., Davis, R.A., Kreiß, J.-P. and Mikosch, T. (eds) Handbook of Financial Time Series, Part 5. Springer, pp. 767-785.

[25] Ratner, M. (1996) 'Investigating the behaviour and characteristics of the Madrid stock exchange', Journal of Banking and Finance, vol. 20, pp. 135-149.

[26] Siourounis, G.D. (2002) 'Modelling volatility and test for efficiency in emerging capital markets: the case of the Athens stock exchange', Applied Financial Economics, vol. 1, pp. 47-55

[27] Wang, Z., Kutan, A. and Yang, J. (2005) 'Information flows within and across sectors in Chinese stock markets', The Quarterly Review of Economics and Finance, vol. 45, pp. 767-780. 